\title{
PARTICIPACIÓN CIUDADANA EN LA ELABORACIÓN DE PRESUPUESTOS CON PERSPECTIVA DE GÉNERO. ESTUDIO DE CASO: MUNICIPIOS DE SONORA ${ }^{69}$
}

Adria Ailev Murillo González

Sumario: Introducción. I.Planteamiento de un problema sobre cultura de la legalidad y participación ciudadana. II. Precisiones del tema en México III.Cuestionamiento cuyas respuestas ayuda a la comprensión del tema. IV. Marco conceptual y antecedentes del Presupuesto Participativo, con Perspectiva de Género. V. Conclusiones

\section{Resumen}

En este documento se examina la cultura de la legalidad al revisar como estudio de caso la medida en la que los municipios del estado de Sonora incorporan la participación ciudadana y la perspectiva de género en la elaboración del Plan Municipal de Desarrollo 2012-2015 y el presupuesto municipal de gastos en 2013, acorde a la normatividad aplicable .

\begin{abstract}
In this document the culture of legality is examined by reviewing the case study:"the extent in which the municipalities of State of Sonora incorporate the citizen participation and the gender perspective in the preparation of the Municipal Plan for Development 2012-2015 and the Municipal Budget of Expenditure in 2013, following the corresponding regulatory provisions.
\end{abstract}

\section{Introducción}

Para cumplir con el objetivo ${ }^{70}$ central del proyecto de investigación final de la asignatura de Teoría general del Derecho, de cuyo tema básico de la Cultura de la Legalidad, seleccioné al inicio del ciclo escolar el apartado "A. Reformas Legislativas en materia de participación ciudadana", y siendo originaria del Estado de Sonora me di a la tarea de investigar al respecto, para elegir un caso relacionado a mi tema, y es así que me puse en contacto con la Academia de Derecho Administrativo del Departamento de Derecho de la

\footnotetext{
${ }^{69}$ Trabajo final de Investigación de la estudiante del tercer semestre de la Licenciatura en Derecho del ITESM Adria Ailev Murillo González para acreditar la asignatura Teoría General del Derecho 70 "reflexionar e investigar sobre las diferentes reformas legislativas que se llevan a cabo en este período de Sesiones del Congreso como una expresión de la cultura de la legalidad y la participación ciudadana como formas de vida democrática"
} 
Unidad Regional Centro de la Universidad de Sonora para preguntar sobre los proyectos que estaban realizando sus académicos ${ }^{71}$. Me remitieron a la página de la Universidad http:// serviciosocial.uson.mx para que revisara cual de los proyectos listados seria de mi interés y darme información adicional a la proporcionada en la página. Una vez hecha la selección, me dieron una serie de lecturas ${ }^{72}$, una lista de documentos y leyes a consultar en los portales como material para mi tarea. Durante la primera parte del ciclo escolar realicé lo sugerido y procedí a ordenar mis fichas bibliográficas además de los materiales obtenidos, por su contenido para redactar el trabajo final. Mil preguntas surgían en mi cabeza, comencé a escribir, borrar, volver a las lecturas, a las fichas bibliográficas sobre el tema, volver a escribir, borrar, escribir varias veces hasta conseguir lo siguiente:

\section{Planteamiento de un problema sobre cultura de la legalidad y participación ciudadana}

\section{¿Para quién? o ¿quiénes?}

1) Para las autoridades municipales del estado de Sonora(Los primeros obligados a cumplir con la legalidad)

\section{¿Porqué?}

Porque la Ley de Participación Ciudadana del Estado de Sonora (2011), establece dentro de los instrumentos de participación ciudadana, el presupuesto participativo, como una metodología a observar (obligación) tanto para el gobierno del estado como para los 72 municipios de la entidad. Por lo tanto, desde el 2012 los presupuestos municipales estuvieron en posibilidad de ser formulados con base en la participación ciudadana (presupuesto participativo) ${ }^{73} \mathrm{y}$, vinculando dicho instrumento con las

\footnotetext{
${ }^{71}$ Alberto Haaz Díaz, María Inés Aragón Salcido, Adria Velia González B, Aleida Guadalupe Valenzuela Miranda,Jesús Miguel Maya Rodríguez

${ }^{72}$ Ver Bibliografía

${ }^{73}$ De conformidad con la Ley de Participación Ciudadana, en su artículo 87, el presupuesto participativo tendrá por objeto:

I.- Propiciar una distribución democrática de los recursos públicos de que disponen los gobiernos estatal y municipales, mediante un mecanismo público, objetivo, transparente y auditable, que posibilita intervenir en la solución de los problemas prioritarios de las comunidades sonorenses;

II.- Efectuar obras prioritarias para la recuperación del espacio público; el mejoramiento y rehabilitación de calles; la rehabilitación o creación de áreas verdes; el mejoramiento o
} 
disposiciones de la Ley para la Igualdad entre Mujeres y Hombres en el Estado (LIMYHESON) ${ }^{74}$ incorporar al presupuesto participativo la perspectiva de género.

2) Para la sociedad

\section{¿Por qué?}

Porque no solo deben participar en la elección de sus autoridades sino también darle seguimiento a su actividad durante el tiempo que duren en el puesto (participación ciudadana) para asegurar que cumplan sus compromisos de campaña y con sus obligaciones del puesto que obtuvieron.

3)Para los encargados de vigilar la actuación de las autoridades(órganos de control : interno-contraloría-dentro del Ayuntamiento o externo el órgano técnico del poder Legislativo el Instituto Superior de Auditoría y Fiscalización)

\section{¿Por qué?}

Porque si se actualizase el supuesto normativo de irregularidades en el ejercicio del gasto se puede aplicar correctivos $o$ las sanciones correspondientes al poder ejecutivo-Ayuntamiento representado por el presidente municipal----.

Como segundo paso elaboré una lista de posibles supuestos (hipótesis) que tendrían que estar detrás del problema planteado y que al final de la investigación en su caso, se comprobaría o no.

construcción de infraestructura cultural, deportiva y recreativa; así como acciones de desarrollo sustentable, fortalecimiento de la seguridad pública y la cultura;

III.- Generar un proceso de democracia directa, voluntaria y universal, que contribuya a fortalecer espacios comunitarios de reflexión, análisis, revisión y solución a los problemas prioritarios, construyendo una ciudadanía consciente y participativa; y

IV.- Establecer un vínculo corresponsable entre el gobierno y los gobernados que permita generar procesos ciudadanos de análisis, programación, vigilancia y control de los recursos públicos.

${ }^{74}$ El artículo14 de la Ley para la Igualdad entre Mujeres y Hombres del Estado de Sonora, dispone que corresponde a los ayuntamientos de los municipios:

I.- Ejecutar la política municipal en materia de igualdad entre mujeres y hombres, en concordancia con las políticas nacional y estatal;

II.- Coadyuvar con el gobierno federal y con el gobierno estatal, en la consolidación de los programas en materia de igualdad entre mujeres y hombres;

III.- Diseñar, formular y aplicar campañas de concientización que promuevan los valores y contenidos de la presente ley;

IV.- Fomentar la participación social, política y ciudadana dirigida a lograr la igualdad entre mujeres y hombres, tanto en las áreas urbanas como en las rurales; $y$

V.- Incorporar en sus Proyectos de Presupuestos de Egresos la asignación de recursos para el cumplimiento de la Política Municipal en Materia de Igualdad entre Mujeres y Hombres. 


\section{Hipótesis}

Las actuales autoridades municipales de Sonora, no han transformado el modelo cerrado actual de planeación,elaboración, ejecución, evaluación y control del presupuesto, en un modelo de gestión pública moderno, abierto y participativo, que les posibilite integrar a tal proceso, la participación ciudadana y el enfoque de género:

a) por inexperiencia(tomaron protesta en el mes de septiembre de 2012), y/o

b) por la falta de asesoría al respecto y/o

c)por la falta de exigencia de los órganos de control( inexperiencia por ser para la mayoría la primera vez que ocupan tales puestos ) y/o

d) por no tener los sonorenses una cultura de la participación y tener poco tiempo en vigor la Ley de participación ciudadana)

Precisiones del tema en México según las lecturas, información proporcionada y consultas realizadas

A casi dos décadas de analizarse en nuestro país el tema del Presupuesto Participativo pro-equidad de género, desde las distintas organizaciones no gubernamentales e institucionales locales y federales solo se cuenta la experiencia del Distrito Federal como la mas acabada ${ }^{75}$ en relación a contar con un presupuesto que incorpore la perspectiva de género en las distintas etapas del proceso presupuestal, que contribuya a reducir las desigualdades de derechos entre mujeres y hombres.

Para tal efecto, los diversos órdenes de gobierno vía el presupuesto de egresos debiesen llevar a cabo sus políticas públicas y resaltar objetivos específicos, como el de la equidad entre géneros. Como lo señalan numerosos estudios, el país ha experimentado progresivamente una feminización de la pobreza, que es necesario y urgente combatir. Para que en México se experimente una auténtica equidad entre géneros, es preciso que las mujeres dispongan de las mismas herramientas de desarrollo y bienestar, que aquellas de las que disponen los hombres.

\footnotetext{
${ }^{75}$ Recientemente ( 23-27 Septiembre de 2013) en Aguas Calientes-México, se le otorgó el primer premio por dicha experiencia en la II Cumbre iberoamericana de Agendas Locales de Género
} 
¿Cómo hacer posible tal pretensión?

Una herramienta adecuada lo son los Presupuestos participativos con Perspectiva de Género, la cual favorece el diseñar políticas y programas, para asignar recursos, que busca la igualdad de oportunidades entre mujeres y hombres. Parte de un análisis que toma en cuenta las necesidades, intereses y perspectivas de diferentes grupos de la sociedad < por medio de la participación en Foros, consulta durante las campañas políticas, por medios electrónicos, entre otros $>$. Esto permite que los que toman decisiones en materia presupuestal municipal estén mejor informados y que el impacto de las asignaciones por ellos realizadas sea más eficiente para el propósito considerado.

Un presupuesto municipal que ignore las desigualdades entre hombres y mujeres necesariamente replicará o mantendrá sin cambiar las relaciones inequitativas de género prevalecientes hasta el día de hoy. Por ello, señalan los expertos que para determinar si el ingreso y gasto público obstaculiza o promueve la igualdad de género, y si los compromisos gubernamentales municipales por la equidad se traducen en compromisos monetarios, es necesario reorganizar las prioridades de los recursos públicos hacia esquemas más equitativos y eficientes de percepción de ingresos y uso de recursos, así como determinar la manera en que las asignaciones presupuestales afectan las oportunidades sociales y económicas de hombres y mujeres.

\section{En un intento de explicar y relacionar lo anterior con mi tarea:}

El panorama en el Estado de Sonora en el que al iniciar el año(2013) prácticamente iniciaron 72 nuevas administraciones municipales ${ }^{76}$ se presenta como una oportunidad inmejorable para el propósito de mi trabajo de investigación sobre la cultura de la legalidad y concretamente en relación con las Reformas Legislativas en materia de participación ciudadana y averiguar sobre el avance de los gobiernos municipales en materia de Presupuesto Participativo con Perspectiva de Género, para el 2013.

\footnotetext{
${ }^{76}$ Dado que durante el 2012 se llevaron a cabo elecciones para renovar entre otras, a las administraciones públicas municipales de los $72 \mathrm{H}$ Ayuntamientos
} 


\section{Objetivo general:}

Mostrar los resultados de la investigación---documental y virtual en la web---, en relación al número de ayuntamientos de los municipios del Estado de Sonora que cumplieron (principio de legalidad) con las obligación de elaborar sus planes municipales de desarrollo y presupuestos con la participación de sus ciudadanos y con perspectiva de género para el 2013.

\section{Marco conceptual y antecedentes del Presupuesto Participativo, con} perspectiva de género, (modalidad interrogativa)

\section{¿Qué es un presupuesto con enfoque de género?}

Un presupuesto con enfoque de género es una de las principales herramientas de la política pública, que permite diseñar, aplicar y evaluar el ejercicio de los recursos desde una perspectiva que reconoce las diferencias existentes por razones de género $y$, en esa medida, permite atender las necesidades diferenciadas de mujeres y hombres. Para clarificar lo anterior se hace necesario acudir al Glosario de términos relacionados con la transversalidad de género ${ }^{77}$, conforme al cual:

1) Enfoque de género es una herramienta metodológica que se utiliza para analizar el impacto diferenciado, en hombres y mujeres, de las políticas y acciones de los gobiernos u organizaciones. Esta herramienta apunta a promover la equidad de género en toda la sociedad, entendiendo como tal a "la distribución justa de recursos y beneficios entre mujeres y hombres conforme a normas y valores culturales."

\section{2)"Sistema sexo-género"}

Sexo y género son categorías distintas que no necesariamente se encuentran asociadas. La noción de sexo hace referencia a las diferencias biológicas que existen entre hombres y mujeres, mientras que el género se refiere a las diferencias socialmente construidas $y$ atribuidas a hombres y mujeres por ejemplo el color rosa es para las mujeres y el azul para los varones, los

\footnotetext{
${ }^{77}$ Glosario de términos relacionados con la transversalidad de género, [en línea], España, Proyecto Equal 2007 [consultado el 20 de septiembre de 2013]

en:http://www.eculturas.com/final/Documenta/texto/GlosarioGenero.pdf
} 
hombres no lloran, las mujeres sí. Nuestra sociedad se construye sobre la base de este "sistema sexo-género" que atribuye roles, obligaciones y derechos de manera diferenciada.

De esta forma se construye una estructura organizacional que determina la forma de vida de las sociedades, definiendo formas de producción, relaciones, estilos de vida y concepciones en torno al empleo, la salud, entre otros. En otras palabras, que el sexo se determina en forma natural, mientras que el género, en tanto construcción social y cultural, es susceptible de determinarse y modificarse en el tiempo y en el espacio.

\section{3) Presupuestos con enfoque de género}

Son aquellos que traducen los compromisos gubernamentales relativos a la equidad de género en compromisos monetarios concretos. Este tipo de presupuestos no son neutros al género, aunque esto no significa que sean presupuestos exclusivos para mujeres. A través de estos presupuestos se impulsa la inclusión transversal del enfoque de género en las políticas y programas nacionales. Este enfoque debe ser incluido en cada una de las etapas del proceso presupuestario. Para llevar a cabo un proceso presupuestario con enfoque de género, es importante tener una visión integral de todo el proceso y admitir las restricciones que se presentan para que puedan ser superadas. Es necesario saber ¿cómo se deciden los presupuestos?, ¿ cuál es la regulación marco de los mismos?, ¿cuales son las partidas presupuestarias? y ¿cómo es que cada una de ellas incide en verdaderas mejoras en términos de equidad?

\section{4) Neutralidad en los presupuestos}

Los presupuestos no son neutros puesto que tienen un impacto diferenciado sobre hombres y mujeres. Si se observan únicamente los gastos e ingresos (números), los presupuestos pueden aparecer como neutros. Por tanto, en el diseño de políticas gubernamentales que involucran la asignación de recursos en la formulación de los presupuestos respectivos, deben tenerse en cuenta las diferencias de género de la población objetivo, considerando por ejemplo el número de mujeres o de niñas y el número de hombres y de niños a quienes 
también se pretende beneficiar con satisfactores u servicios diferenciados como por ejemplo canchas de football en vez de academias de danza o talleres de carpinterías en vez de salas de belleza o de costura.

\section{5) "Transversalización" del enfoque de género}

Las construcciones sociales sobre los roles que deben desempeñar los hombres y las mujeres atraviesan todas las actividades humanas, incluidas las relativas a la definición de las políticas públicas. La incorporación del enfoque de género en las políticas públicas apunta a "hacer que las preocupaciones y las experiencias de las mujeres y los hombres sean un elemento central de la elaboración, puesta en práctica, supervisión y evaluación de políticas y programas, con el fin de que ambos grupos se beneficien y se impida que la desigualdad se reproduzca."

Lo anterior implica que la búsqueda de la equidad entre hombres y mujeres se encuentre presente en todas las estrategias del gobierno y que tanto los hombres como las mujeres participen no solamente en la implementación de los programas o proyectos, sino a lo largo de todo el proceso presupuestario, incorporando sus intereses, necesidades y prioridades.

De lo descrito, surge el siguiente cuestionamiento cuyas respuestas ayudan a la comprensión del tema ¿Por qué abogar por que se cumpla con la normatividad del caso que nos ocupa?

- Por construir en nuestro medio, día a día la cultura de la legalidad

- Por construir en nuestro medio la cultura de la participación

- Por el beneficio mutuo de hombres y mujeres.

- Porque las estructuras (sistema sexo-género) diferenciadas e injustas, establecen una distribución entre hombres y mujeres, del trabajo, los recursos, el tiempo, los quehaceres y derechos, determinando una situación de subordinación femenina generalizada. Por ejemplo, en los países en vías de desarrollo las mujeres perciben salarios que en promedio equivalen al $73 \%$ 
de los percibidos por sus contrapartes masculinas; en los países industriales esta cifra llega al $77 \%{ }^{78}$

- Porque la introducción del enfoque de género no sólo contribuye a alcanzar mayores niveles de equidad (una sociedad más justa) sino también mayores niveles de eficiencia en lo que respecta al impacto de las políticas públicas. Diferentes estudios presentan pruebas que sustentan el postulado de que "a mayor equidad, mayor eficiencia", este es el caso de un estudio que indica que al eliminar las desigualdades del mercado laboral latinoamericano se incrementaría el ingreso de las mujeres en un $50 \%$ y el ingreso nacional en un $5 \% .{ }^{79}$

- Porque el municipio es considerado uno de los espacios de gobierno en donde la presencia de las mujeres desempeña un papel cada vez más estratégico para marcar el rumbo hacia un desarrollo económico y social mucho más equilibrado, incluyente y sustentable.

- Porque es importante luchar para que en el mundo haya una mayor presencia de las mujeres en la toma de decisiones políticas y económicas.

\section{Antecedentes}

a) La experiencia Internacional. Toda propuesta que pretenda favorecer el presupuesto participativo con perspectiva de género, debe acudir a las experiencias que se han estado implementando en diversos países. Las experiencias en presupuestos participativos han acumulado enseñanzas interesantes y se están expandiendo en diferentes países de América Latina. Hay un conjunto de iniciativas, especialmente municipales, ya sea en municipios provinciales ya en pequeños cantones o municipios distritales, algunos elevan una mirada al presupuesto nacional. Varias experiencias no son tan recientes, como la pionera del Municipio de Porto Alegre, Brasil,

\footnotetext{
${ }^{78}$ Encina Jenny, Chile Crece Contigo: El efecto de la implementación de Salas Cunas Públicas en Participación Laboral Femenina, Pobreza y Distribución de Ingresos http://www.pnud.cl/areas/ReduccionPobreza/2012/2008_1.pdf [consultado el 20 de Septiembre de 2013]

79 Encina Jenny, op. cit

Félix Guarocuya, et. al,La Desigualdad Social y los Retos Financieros

http://www.pucmm.edu.do/RSTA/Academico/viii/centros/cueps/Publicaciones/Documents/LaDesigualdadSocia lylosRetosFinancieros[Integro].pdf [consultado el 23 de octubre de 2013]
} 
capital del estado de Río Grande do Sul(1 millón 300 mil habitantes) que dio inicio a la experiencia participativa respecto a los presupuestos en 1989, para lo cual abrió procesos de consulta en su jurisdicción con diferentes grupos sociales y con el fin de discutir y definir las prioridades en las inversiones y los gastos de los presupuestos, sustentados en criterios objetivos aceptados por la comunidad.

En lo que concierne a los presupuestos con perspectiva de género, la iniciativa de impulsarlos “... responde a una constatación dramática para las mujeres: el impacto diferencial y negativo de presupuestos supuestamente neutrales al género, en un contexto donde la "neutralidad" de las relaciones de género está muy lejos de lograrse. Por lo mismo, son más bien presupuestos "ciegos al género" pues ignoran los roles socialmente determinados y diferenciados, y las consiguientes responsabilidades y capacidades también diferenciadas de mujeres y hombres. Estas diferencias están estructuradas de tal forma que dejan a la mujer en posición desigual respecto al hombre, con menor poder político, social y económico en su comunidad y en la sociedad" 80

Se considera que un impulso fundamental a este interés creciente han sido las conferencias y cumbres mundiales de la década de los 90 , especialmente la de Beijing. Estas conferencias han levantado recomendaciones claras respecto a la importancia de que las mujeres incursionen en los espacios de decisión y/o generen mecanismos de participación que logren influir en las políticas gubernamentales. Una recomendación constante, pocas veces cumplida por los gobiernos, ha sido justamente el contar con "recursos necesarios y suficientes". De allí que algunas iniciativas se sustentan con mayor énfasis en algunas de estas conferencias: la de El Cairo sobre Población (1994), en el caso de una de las experiencias mexicanas; la IV Conferencia Internacional sobre la Mujer, en Beijing (1995), que parece ser base de todas las demás, en la medida que la Plataforma de Acción plantea

\footnotetext{
${ }^{80}$ Elson, Diane.Seguimiento de los Presupuestos Gubernamentales para el Cumplimiento de la CEDAW. Informe para UNIFEM. Department of Sociology, University of Essex, UK/ Levy 2005, Economic Institute, Bard College, USA.
} 
explícitamente que "se integre una perspectiva de género en las decisiones presupuestarias", y la Cumbre de Copenhague sobre Desarrollo (1995) aportó recomendaciones y perspectivas en ese sentido.

El trabajo en presupuestos desde la perspectiva de género es una de las puertas más importantes para comenzar a llevar la pelea democrática al terreno de los procesos macroeconómicos y macro políticos. Por algo, una de las peleas más fuertes en la Sesión Especial de Beijing (Nueva York, junio 2000) fue justamente respecto a la negativa de muchos gobiernos latinoamericanos y del mundo, a comprometer medidas para valorar y reflejar la contribución de las mujeres en los presupuestos nacionales.

Proponer un presupuesto con sensibilidad de género, como lo define el Commonwealth que ha lanzado esta iniciativa hace ya algunos años, con resultados como el análisis presupuestal con contenido de género en varios de los países miembros (entre ellos, los más exitosos son los de Sudáfrica y Australia), es proponer una metodología y un conjunto de herramientas diseñadas para facilitar la aplicación de un análisis de género en la formulación de los presupuestos de gobierno y en el destino de los recursos presupuestales. El objetivo de tal iniciativa es aumentar las políticas y medidas fiscales y proveer de un mecanismo para determinar su impacto en mujeres, hombres, niñas y niños. Por su parte, Budlender, Debie y Sharp, señalan que los presupuestos sensibles al género no son presupuestos separados para mujeres sino que más bien buscan la incorporación de esta perspectiva en todos los aspectos de la elaboración del presupuesto en los niveles nacionales y locales; promueven un compromiso activo y la participación de grupos de mujeres (y hombres sensibles) que monitorean y evalúan los gastos $y$ las rentas gubernamentales desde un enfoque de género; promueven también el uso más efectivo de los recursos para lograr tanto la equidad de género como el desarrollo humano, enfatizan la elaboración de prioridades en vez del incremento del gasto público y destacan la reorientación de los programas 
dentro de sectores en vez de cambios de las cantidades generales asignadas a sectores específicos ${ }^{81}$

De lo anterior los expertos concluyen que el análisis y el desarrollo de los presupuestos participativos con perspectiva de género son un hecho político y político-económico y no solo técnico. No hay una sola forma o un solo modelo para acercarse a esta tarea. Sus posibilidades varían de acuerdo con: 1) las formas específicas en que los presupuestos son diseñados, 2) la extensión y contenido democrático de los procesos de descentralización,3) grado de apertura que los gobiernos nacionales y municipales tengan con respecto a la participación ciudadana, así como 4) la densidad y capacidad de exigencia de las sociedades civiles, incluyendo los movimientos de mujeres. En América Latina ya podemos encontrar varias iniciativas. Aunque todavía desconectadas entre sí, han comenzado en este nuevo siglo a reconocerse y alimentar una red informal que comparte avances y dificultades. En el ámbito federal, local y municipal se han desarrollado estrategias diversas, lo común, sin embargo, salvo la experiencia del D.F., ha sido el asumir un enfoque de derechos y un enfoque democrático, alimentados por la voluntad política de empoderar a las mujeres, ante ellas y ante la sociedad y el Estado.

El sentido de las estrategias también coincide: todas buscan desarrollar herramientas y entendimientos sobre género y presupuestos, así como las habilidades y reflexiones de las mujeres, tanto para argumentar a favor de estos presupuestos como para exigir y/o facilitar mecanismos de transparencia y rendición de cuentas. Buscan también incidir en mujeres (y hombres) líderes, ya sea en cargos públicos ya sea en organizaciones de la sociedad civil. Favorecen el fortalecimiento de las dinámicas organizativas de las mujeres y buscan ampliar los espacios para posicionar un sentido de derechos y responsabilidades de la ciudadanía, particularmente las mujeres, frente a la exigencia de transparencia, rendición de cuentas y gobernabilidad

\footnotetext{
${ }^{81}$ Budlender, Debie y Sharp, Cómo realizar un análisis de presupuesto sensible al género. Secretaría de La Mancomunidad Británica. Rhonda (2000). [ Disponible en: http://presupuestoygenero.net/Herramientas/H028BudlenderGL.pdf]
} 
democrática. Entre los países donde ya se está desarrollando este análisis se encuentran los de la región andina (Perú, Bolivia, Ecuador y Colombia), además de México, Chile, Honduras, Chile, Argentina.

\section{b) Síntesis de la experiencia en México}

Necesario mencionar a, Equidad de Género y FUNDAR, dos instituciones que están impulsando, coordinadamente, el proyecto "Fortaleciendo la incidencia de mujeres líderes en presupuestos públicos desde el enfoque de género", apoyadas por UNIFEM-México y Centroamérica, y otras agencias. Explícitamente se sustentan en el Plan de Acción de la Conferencia de $E$ Cairo que señala que en los países en desarrollo se deben destinar recursos nacionales "que cubran dos tercios del total de la plata requerida para programas enfocados en desarrollo humano con el consiguiente fortalecimiento de la ciudadanía y del goce de derechos".

Una segunda experiencia es la asumida por la Comisión Nacional de la Mujer, al nivel del gobierno del Distrito Federal. Su objetivo es identificar las inequidades que existen, desde el proceso de programación hasta la aplicación de los recursos financieros, en programas y proyectos que beneficien a la población femenina, y hacer conciencia al respecto entre los servidores de las dependencias e instituciones de la administración pública federal. Es un programa multisectorial que requiere también a organizaciones de la sociedad civil. Proponen la incorporación del enfoque de género, como metodología de trabajo por cada uno de los sectores en sus tareas de planificación y programación, identificando inequidades, determinando las causas y las acciones para combatirlas".

"El Instituto Nacional de las Mujeres (Inmujeres), con el propósito de cumplir con sus objetivos y atribuciones creó en el 2005 el Fondo de Inicio y Fortalecimiento para las Instancias Municipales de las Mujeres, a través del cual se promovía el inicio y fortalecimiento de Instancias Municipales de las Mujeres (IMM) con el fin de trabajar a favor de la no discriminación, la igualdad de oportunidades y trato entre géneros, así como el ejercicio pleno de los 
derechos de las mujeres y su partición equitativa en la vida política, cultural, económica y social del país.

El fondo bajo este concepto operó durante 3 años $(2005,2006,2007)$, período durante el cual se benefició a 227 municipios.

\section{c) Marco jurídico y ámbito local (Experiencia de Caso: Sonora)}

A partir del 2008 se reformula la estrategia del Fondo de Inicio y Fortalecimiento para las Instancias Municipales de las Mujeres, dando especial importancia al ámbito local como parte primordial para lograr una transformación en las políticas públicas de los municipios que posibiliten el desarrollo local incluyente. Con este propósito se crea el Fondo para Desarrollo de las Instancias Municipales de las Mujeres (FODEIMM) ${ }^{82}$. Y dentro del marco jurídico, en el presupuesto de egresos de la federación, se incorpora, el CAPÍTULO IV, De la perspectiva de género ${ }^{83}$ y en el DOF 19-

\footnotetext{
${ }^{82}$ Centro de Estudios para el Adelanto de las Mujeres y la Equidad de Género .Mtra. María de los Ángeles Corte Ríos. Directora General

${ }^{83}$ PRESUPUESTO DE EGRESOS DE LA FEDERACIÓN PARA EL EJERCICIO FISCAL 2012.CÁMARA DE DIPUTADOS DEL H. CONGRESO DE LA UNIÓN. Secretaría General. Secretaría de Servicios Parlamentarios. Dirección General de Servicios de Documentación, Información y Análisis. Nuevo Presupuesto DOF 12-12-2011. CAPITULO IV

De la perspectiva de género

Artículo 28. En cumplimiento a la Ley General para la Igualdad entre Mujeres y Hombres, el Ejecutivo Federal impulsará la igualdad sustantiva entre mujeres y hombres a través de la incorporación de la perspectiva de género en el diseño, elaboración, aplicación, seguimiento y evaluación de resultados de los programas de la Administración Pública Federal. Para tal efecto, las dependencias y entidades deberán considerar lo siguiente:

I. Incorporar la perspectiva de género y reflejarla en la matriz de indicadores para resultados de los programas bajo su responsabilidad;

II. Identificar y registrar la población objetivo y la atendida por dichos programas, diferenciada por

sexo, grupo de edad, región del país, entidad federativa, municipio o demarcación territorial del Distrito Federal, y población indígena en los sistemas que disponga la Secretaría de Hacienda y Crédito Público y en los padrones de beneficiarias y beneficiarios que corresponda;

III. Fomentar la perspectiva de género en el diseño y la ejecución de programas en los que, aun

cuando no estén dirigidos a mitigar o solventar desigualdades de género, se pueda identificar de forma diferenciada los beneficios específicos para mujeres y hombres;

IV. Establecer o consolidar en los programas bajo su responsabilidad, las metodologías de evaluación y seguimiento que generen información relacionada con indicadores para resultados con perspectiva de género, $y$

V. Aplicar la perspectiva de género en las evaluaciones de los programas, con los criterios que emitan el Instituto Nacional de las Mujeres, la Secretaría de Hacienda y Crédito Público y el Consejo Nacional de Evaluación de la Política de Desarrollo Social.

Las acciones contenidas en las fracciones anteriores serán obligatorias en lo relativo a los programas y acciones incorporadas en el Anexo 10 del presente Decreto y para los demás programas federales que correspondan.

Todas las dependencias y entidades ejecutoras del gasto federal que manejen programas para mujeres y la igualdad de género, así como las entidades federativas y municipios que reciban recursos etiquetados incluidos en el Anexo 10 de este Decreto deberán informar sobre los resultados de los mismos, los publicarán y difundirán para darlos a conocer a la población e informarle, en su lengua, sobre los beneficios y requisitos para acceder a ellos, en los términos de la normativa aplicable.
} 
01-2012, se publicaron reformas a la Ley Federal de Presupuesto y Responsabilidad Hacendaria en sus artículos $2^{\circ}$. III Bis.; 23; 27; 28; 41; 58; 107; 110. Asimismo en el Estado de Sonora se publicó la Ley de Participación Ciudadana (LPCESON) y se instituye el instrumento de Presupuestos Participativos. Contemplándose por su parte en la Constitución Política del Estado de Sonora, en la Ley de Gobierno y Administración Municipal (LGAM) y Ley de Planeación del Estado de Sonora (LPESON) diversas disposiciones relativas a la constitución y operación de los Comités de Planeación Municipal y a la determinación de mecanismos de actualización, instrumentación, control y evaluación del Plan Municipal de Desarrollo. ${ }^{84} \mathrm{Y}$ en términos arriba citados lo dispuesto en la Ley para la Igualdad entre Mujeres y Hombres del Estado de Sonora.

\section{Sintetizando la anterior información para el propósito del presente trabajo:}

Para el año 2013 de acuerdo a las normas jurídicas precitadas(legalidad), la presupuestación municipal en el estado de Sonora tendría que basarse en los

\footnotetext{
Para el seguimiento de los recursos destinados a las mujeres y a la igualdad de género, todo programa federal que contenga padrones de beneficiarias y beneficiarios, además de reflejar dicho enfoque en su matriz de indicadores para resultados, generará información de manera desagregada, al menos por sexo y entidad federativa.

Las dependencias y entidades con presupuesto asignado dentro del Anexo 10, que realicen estudios y generen bases de datos o levantamientos de encuestas, deberán hacer públicos sus resultados en sus portales institucionales con el propósito de poder realizar evaluaciones y análisis posteriores. Las dependencias y entidades responsables de la coordinación de los programas contenidos en el Anexo 10del presente Decreto informarán trimestralmente a través del sistema de información desarrollado por la Secretaría de Hacienda y Crédito Público, y en el sistema de Evaluación de Desempeño en los términos y plazos establecidos en las disposiciones respectivas, sobre los aspectos presupuestarios de los programas y los resultados alcanzados en materia de mujeres e igualdad de género, medidos a través delos indicadores y sus metas contenidos en la matriz respectiva. Asimismo, se detallarán los aspectos porcada programa presupuestario, contenido en el Anexo mencionado, la población objetivo y atendida, los indicadores utilizados, la programación y el avance en el ejercicio de los recursos.

${ }^{84}$ (Constituir y operar el Comité de Planeación Municipal de ---------. COPLAM, Artículo 61, II, inciso B, LGAM. Elaborar y aprobar y publicar en los términos de Ley, el Plan Municipal de Desarrollo correspondiente a su periodo constitucional de gobierno y derivar de éste, los programas para la ejecución de obras y la prestación de los servicios de su competencia. Artículos 25-C y 136, II, Constitución Local; 61, fracción II, inciso B LGAM; artículos 8, a nivel municipal, incisos 9, b) y c), 25 de la Ley de Planeación del Estado de Sonora; artículo 87 de la Ley de Participación Ciudadana y el artículo14 de la Ley para la lgualdad entre Mujeres y Hombres del Estado de Sonora. Determinar mecanismos de actualización, instrumentación, control y evaluación del Plan Municipal de Desarrollo. Artículo 61, II, inciso C LGAM y artículos 8, a nivel municipal, incisos 9, b) y c), 25 de la Ley de Planeación del Estado de Sonora.
} 
respectivos Planes Municipales de Desarrollo y elaboración de los Presupuestos de Egresos con participación ciudadana y perspectiva de género.

\section{Metodología}

\section{De la revisión en la red se encontró:}

1) Solo 13 de los 72 municipios cuentan con un portal que proporciona a los visitantes virtuales información de su interés(Agua Prieta, Caborca, Ciudad Obregón, Empalme, Guaymas, Hermosillo, Magdalena de Kino, Navojoa, Nogales, Puerto Peñasco, San Ignacio Río Muerto, San Luis Río Colorado, Santa Ana)

2) Solo cuatro municipios (Agua Prieta, Hermosillo, Navojoa, Puerto Peñasco) cuentan con la publicación en su Portal de su Plan Municipal de Desarrollo.

3) Todos excepto uno de los municipios (Rosario de Tesopaco) cuentan con la publicación en el Portal del Congreso Local de su Ley de Presupuesto de Ingresos.

4) De la revisión de los Portales se desprende que solo el municipio de Hermosillo realizó un ejercicio de participación ciudadana(consulta electrónica) en la elaboración de su Plan Municipal de Desarrollo ${ }^{85}$ y aplicó incipientemente en forma transversal la perspectiva de género

85 Ver en el blog http://haaz-calidad.blogspot.mx de que manera la Secretaría de Hacienda del gobierno estatal de Sonora, trata de difundir el "Presupuesto Ciudadano 2013". Ahí de manera gráfica --dibujos--puede conocerse la distribución del presupuesto de egresos en diversas clasificaciones, como la relativa a los "ejes rectores" del plan estatal de desarrollo. 


\section{Trànsversalización de la Perspectiva de Cénero en Programas Presupuestaríos}

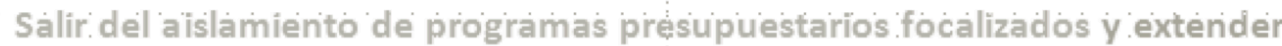
el enfoque a todos los campos de la Administración Pública, no solo a las oficinas especializadas en las mujeres (como el Instituto Sonorense de la Mujer (ISM) o la Dirección de Atención a la Mujer en un Municipio.

\begin{tabular}{|c|c|c|c|c|c|c|}
\hline $\mathrm{O}, \mathrm{P}$. & Fom. Eco. & DAM & $\ldots$ & Tesor. & $\ldots$ & \\
\hline $\begin{array}{l}\text {.... Infraes... } \\
\text { tructura }\end{array}$ & $\begin{array}{l}\text { Financia } \\
\text { miento }\end{array}$ & Ascrorías & $\begin{array}{c}\text { Capacita } . . \\
\text { ción }\end{array}$ & $\begin{array}{c}\text { Estímulo } \\
\text { fiscal }\end{array}$ & $\begin{array}{c}\text { Asistencia } \\
\text { técnica }\end{array}$ & $\begin{array}{l}\text { Igualdad } \\
\text { entre }\end{array}$ \\
\hline$\$ 180$ & $\$ 79$. & $\$ 38$. & $\$ 52$ & $\$ 10$ & $\$ 33$ & \\
\hline
\end{tabular}

De la información proporcionada por los investigadores(as) adscritas a la Academia de Derecho Administrativo (ADA) Se encontró que :

5) A partir de la propuesta del grupo de investigación de la ADA presentada a los recientes estrenados presidentes municipales de Sonora que acudieron al Seminario Presupuesto Participativo con Perspectiva de Género en Municipios $^{86}$ se logró entre otros el objetivo de desarrollar y fortalecer las capacidades de los participantes para incorporar la participación ciudadana y la perspectiva de género en la elaboración del Plan Municipal de Desarrollo 2012-2015 y del presupuesto municipal de egresos 2013 y posteriores, atendiendo las disposiciones normativas correspondientes. Metas alcanzadas: El compromiso de uno de los Ayuntamientos de los 12 Municipios participantes, el de Bacoachi,(periodo constitucional del 16 de septiembre de

86 Actividad contemplada en el Proyecto de Vinculación "El presupuesto participativo con perspectiva de género en el ámbito municipal de Sonora, como herramienta para contribuir a mejorar las administraciones públicas municipales." 
2012 al 15 de septiembre de 2015), para elaborar los presupuestos participativos de 2014 y 2015 con participación ciudadana y perspectiva de género; y para que en el transcurso de la ejecución de su plan y presupuestos, se pueda verificar, medir y evaluar los avances y resultados, utilizando indicadores de desempeño que permitan apoyar decisiones para la mejora de la gestión pública correspondiente (futuro próximo) a demostrar en los informes mensuales .

6)“Exceptuando al de Hermosillo, de los 71 restantes municipios, el de Bacoachi ${ }^{87}$ considera en su Plan Municipal de Desarrollo, que la participación ciudadana se toma en cuenta al consultar sus necesidades durante la campaña política (y dado que hubo renovación en el 2012 de los poderes ejecutivos en los 72 municipios y el Poder legislativo local) se puede considerar válida para los demás municipios tal afirmación, lo mas parecido a participación ciudadana durante las campañas electorales.

7) Los 72 municipios de Sonora no avanzaron para el ejercicio fiscal (2013) en el desarrollo de presupuestos participativos con perspectiva de género como herramienta para contribuir a mejorar sus administraciones públicas municipales. Y en consecuencia, dejaron pasar la oportunidad de:

- Analizar con perspectiva de género situaciones de hecho del ámbito municipal en Sonora que les permita reconocer que la vida de mujeres y hombres puede modificarse en la medida en que no está "naturalmente" determinada, si se toma en cuenta que las diferencias entre unas y otros se dan, más que por su determinación biológica, por las diferencias culturales asignadas a los seres humanos.

- Proponer y diseñar políticas públicas que eliminen las desigualdades en las oportunidades que hoy por hoy se brindan a las mujeres y los hombres de Sonora.

- Hacer visibles a las mujeres como sujetos potenciales del desarrollo, superando las visiones parciales que las consideran "grupos vulnerables" o ciudadanas de segunda categoría.

- conocer que la importancia de la aplicación de la perspectiva de género en la elaboración de los presupuestos radica en las posibilidades que brinda para advertir cómo se produce la

\footnotetext{
$87 \mathrm{http} / / /$ haaz-calidad.blogspot.mx Democracia es igualdad; y presupuesto participativo con perspectiva de género
} 


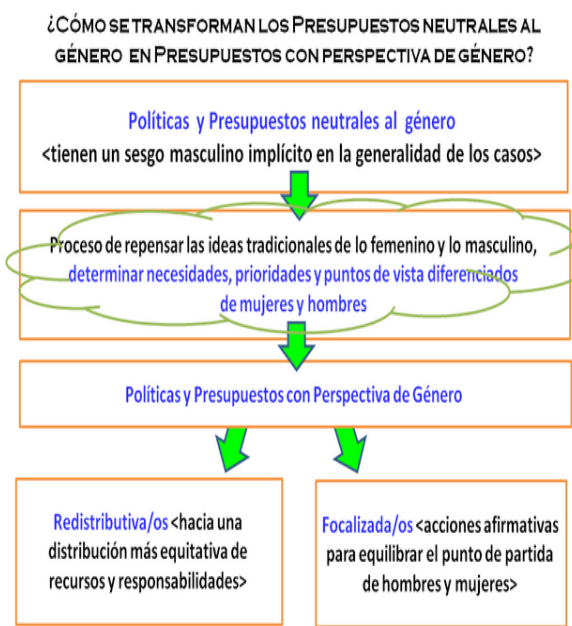

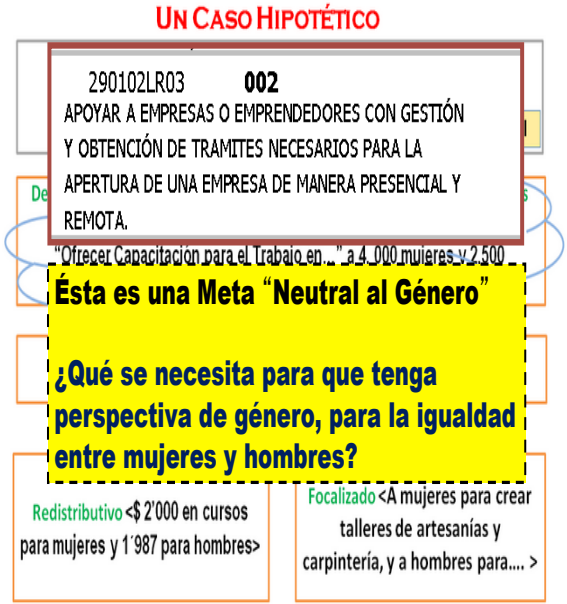

\section{Conclusiones}

Por lo anterior se confirma mi suposición (hipótesis) de que las actuales autoridades municipales de Sonora, no integraron en la elaboración de sus presupuestos, la participación ciudadana y el enfoque de género, a saber:

- por inexperiencia(tomaron protesta en el mes de septiembre de 2012), y/o

- por no buscar o no aceptar asesoría al respecto y/o

- por la falta de exigencia -- insuficiente cultura de la legalidad--,no hay constancia documental en las páginas web de los órganos de control interno y externo en relación a ello,-- inexperiencia por ser para la mayoría la primera vez que ocupan tales puestos--.

- por parte de la sociedad, por no tener una cultura de la participación además del poco tiempo en vigor de la Ley de participación ciudadana (2011), por lo que se debería insistir por los organismos facultados, los académicos y la sociedad en general para que además de los Ayuntamientos de los 72 municipios, el propio Estado de Sonora los próximos ejercicios fiscales 2014 y 2015 elaboren presupuestos con enfoque de género y participación ciudadana que les auxilie en la meta de alcanzar la igualdad social, al

\footnotetext{
${ }^{88}$ como por ejemplo 1)I a redistribución equitativa de las actividades productivas y reproductivas entre los sexos; 2)la justa valoración de los distintos trabajos que realizan mujeres y hombres, especialmente los relacionados con la crianza de los hijos e hijas, el cuidado de personas enfermas y las tareas domésticas; 3) la modificación de las estructuras sociales, mecanismos, reglas, prácticas y valores que reproducen la desigualdad; así como 5) el fortalecimiento del poder de gestión y decisión de las mujeres.
} 
permitir visualizar de manera objetiva el valor de los recursos destinados a hombres y mujeres, niñas y niños, y con ello evaluar las políticas públicas.

\section{¿Cuáles son los retos que enfrentan los Ayuntamientos para la inclusión del enfoque de género en el proceso presupuestario municipal?}

- Los expertos dicen que un primer aspecto lo es la complejidad de lograr la participación ciudadana en la elaboración del presupuesto; un segundo aspecto los tiempos poco oportunos en los que, a veces, son presentados éstos; un tercero, la falta de capacitación del personal de la administraciones municipales en cuanto a temas de género y derechos humanos en general; y uno muy importante los procesos de descentralización de recursos, ya que si bien las decisiones se transfieren al poder municipal, no siempre reciben los recursos de la federación o de los Estados en los tiempos presupuestados para cumplir con los compromisos adquiridos; y uno mas es que la mayoría de los municipios según los investigadores no cuentan con información oportuna y/o adecuada para el análisis con enfoque de género(recolección de estadísticas, diseño de indicadores socioeconómicos tomando en cuenta el género etc.)

\section{Bibliografía y Webgrafía:}

Aguilar, Lorena, Itzá Castañeda, Hilda Salazar y Jacqueline Siles, En búsqueda del género perdido. Equidad en áreas protegidas, Unión Mundial para la Naturaleza/Absoluto,San José Costa Rica, 2002.

Beltrán, Elena y Virginia Maquieira (eds.), Feminismos: debates teóricos contemporáneos, Alianza Editorial, Madrid, 2001.

Budlender, Debie y Sharp, Rhonda (2000). Cómo realizar un análisis de presupuesto sensible al género. Secretaría de La Mancomunidad Británica. http://presupuestoygenero.net/Herramientas/H028BudlenderGL.pdf]

Elson, Diane.Seguimiento de los Presupuestos Gubernamentales para el Cumplimiento de la CEDAW. Informe para UNIFEM. Department of Sociology, University of Essex, UK/ Levy 2005, Economic Institute, Bard College, USA. Montecinos, Egon. El Presupuesto Participativo en América Latina. ¿Complemento o subordinación a la democracia representativa? Publicado en la Revista del CLAD Reforma y Democracia. No. 44. (Jun. 2009). Caracas. http://www.clad.org/portal/publicacionesdelclad/revistacladreformademocracia/ articulos/044-junio-2009/montecinos

Fondo de Desarrollo de las Naciones Unidas para la Mujer (2006). Los presupuestos sensibles al género en la práctica, manual de capacitación. http://www.unfpa.org/webdav/site/global/shared/documents/publications/2006/ gender_manual_pdf ] 
Glosario de términos relacionados con la transversalidad de género, [en línea], España, Proyecto Equal 2007 [consultado el 26 de septiembre de 2012 http://www.eculturas.com/final/Documenta/texto/GlosarioGenero.pdf

Glosario de términos sobre género, Centro Nacional para el Desarrollo de la Mujer y la Familia. Gobierno de los Estados Unidos Mexicanos-Presidencia de la República, Plan Nacional de Desarrollo 2001-2006, México, 2001.

Sistema Nacional para el Desarrollo Integral de la Familia, La perspectiva de género: una herramienta para construir equidad entre mujeres y hombres, DIF, México,1997.

Gobierno del Distrito Federal Presupuesto Ciudadano.

http://presupuesto.atomointeractive.com.mx/conoce/el-presupuesto-

participativo-en-mexico.html

Gobierno del Estado de Sonora

--“Ley de Gobierno y Administración Municipal"

http://www.cgeson.gob.mx/servicios/leyes/estatal/leyes/Ley\%20075\%20De\%2

0Gob\%20y\%20Admon\%20Municipal.pdf]

--“Ley de Participación Ciudadana del Estado de Sonora"

http://gobierno.sonora.gob.mx/documentos/ --"Ley para la Igualdad entre Mujeres y Hombres en Sonora"

http://cedoc.inmujeres.gob.mx/ftpg/Sonora/son02.pdf

--"Ley de Planeación para el Estado de Sonora"

http://transparencia.esonora.gob.mx/NR/rdonlyres/2C1DE41D-E4E1-4887-

871B-D4705CDE8533/74595/Ley040DePlaneacion.pdf

--“Reglamento del COPLADES", http://docs.google.com/leaf?id=

--"Ley de presupuesto de egresos, contabilidad gubernamental y gasto público del Estado de Sonora",

http://www.secsonora.gob.mx/ocda/uploads/wfd_121563301748751679d80d2-ley_de_presupuesto_y_cg.pdf]

Instituto Latinoamericano y del Caribe de Planificación Económica y Social ILPES (2004). Metodología del Marco Lógico.

http://medusa.coneval.gob.mx/cmsconeval/rw/resource/coneval/eval_mon/132 2.pdf?view=true]

Secretaría de Hacienda y Crédito Público. Sistema de Evaluación del Desempeño. [Disponible en: http://www.funcionpublica.gob.mx/index.php/uegdg/sistema-de-evaluacion-deldesempeno.html]

Virginia Vargas Valente Presupuestos sensibles al género: las experiencias en América Latina. http://www.unifemandina.org/docu.html 